(Aus dem physiologischen Institut der k. k. Universität Lemberg.)

\title{
Über Erregbarkeit der Grosshirnrinde und Auslösbarkeit von Rindenepilepsie unter Einfluss von Schlafmitteln wie nach Verabreichung grösserer Bromgaben ${ }^{1}$ ). Von
} Prof. Dr. G. Bikeles und Dr. L. Zbyszewski.

Zweck dieser Untersuchungen war die Eruierung des Verhaltens der Erregbarkeitsschwelle wie der Summationsfähigkeit behufs Erzeugung von Jacks on' scher Epilepsie beim Hunde unter Einwirkung sei es verschiedener Schlafmittel, sei es grösserer Bromgaben. Von Schlafmitteln kamen zur Anwendung: Chloralhydrat, Amylenhydrat, Dormiol, Natriumveronal, Luminalnatrium, Adalin und Bromural, im ganzen bei 30 Versuchstieren.

Brom gebrauchten wir ausschliesslich als Natriumsalz, und zwar, sei es per os, sei es als Injektion in die Vena cruralis. Brom als einmaljge Gabe wurde bei 14 Versuchstieren verabreicht; bei drei derselben wurde zuvor durch $6-9$ Tage kochsalzfreie Nabrung gegeben. Ausserdem wurde bei drei anderen Tieren Bromnatrium durch eine Reihe von Tagen (von 5-9) bei gleichzeitiger Kochsalzentziehung verabreicht.

Das Versuchsverfahren war im allgemeinen folgendes: Zunächst wurde beim Hunde die psychomotorische Region der Hirnrinde auf der einen Seite blossgelegt und die Erregbarkeitsschwelle für die hintere und vordere Extremität, eventuell auch für den Orbicularis oculi festgestellt. Dann wurde die Region für die hintere Extremität (in einer Minderheit der Fälle die Region für den Augenschliesser und nur ganz ausnahmsweise die Gegend der vorderen Extremität) sukzessive nach entsprechenden Pausen mit anwachsenden Stromstärken durch eine jeweilige konstante Anzahl von Sekunden (gewöhnlich 30) bis zum Erscheinen eines Anfalls von Rindenepilepsie gereizt. Es sei hier gleich bemerkt, dass in allen Versuchen, in

1) Vorgelegt der Akademie der Wissenschaft. Krakau, am 8. Juni 1914. Erscheint in polnischer Sprache.

Pflüger's Archiv für Physjologie. Bd. 158. 
denen nicht ausdrïcklich die Erzeugung des Anfalles als Folge der Reizung einer anderen Region namhaft gemacht wird, die Reizung in der Gegend der hinteren Extremität statthatte.

Nach der auf diese Weise erfolgten Feststellung der Erregbarkeitsschwelle und der Reaktionsweise des betreffenden Tieres in Form eines Anfalles wurde dem Hunde, sei es eines der erwähnten Schlafmittel und dann in der Regel per os, mittels Sonde, sei es Natriumbromat bald per os, bald per venam verabreicht. In allen Fällen einer Verabreichung per os wurde eine entsprechende Menge von (bei gewissen Hypnotika warmer) Flüssigkeit eingeführt. Nach Ablauf einer entsprechenden Zeit wurde nun abermals das Verhalten der Grosshirnrinde bezüglich Erregbarkeit und Auslösbarkeit von Rindenepilepsie studiert.

Die auf diese Weise erhaltenen Ergebnisse veranschaulichen die einzelnen beigefügten Tabellen.

(Siehe die Tabellen I-IX auf S. 237-247.)

Daraus ersieht man vor allem, dass eine Herabsetzung der Erregbarkeitsschwelle und die Aufhebung der Fähigkeit zur. Erzeugung von J a ck s o n' scher Epilepsie nicht immer einander ganz entsprechen. Die Erregbarkeitsschwelle wurde in unseren Versuchen recht auffallend herabgesetzt unter der Einwirkung von Amylenhydrat und Dormiol, durch Amylenhydrat eigentlich noch mehr als durch Dormiol.

So sinkt für die hintere Extremität unter Einwirkung von 7 cem Amylenhydrat bei drei Versuchstieren die Erregbarkeitsschwelle einmal von 180 auf $135 \mathrm{~mm}$ R.-A., beim zweiten Hund von 170 auf $125 \mathrm{~mm} \mathrm{R.-A.,} \mathrm{beim} \mathrm{dritten} \mathrm{Hund} \mathrm{von} 140$ auf $90 \mathrm{~mm}$ R.-A. Auch nach $6 \mathrm{ccm}$ Amylenbydrat wird die Erregbarkeitsschwelle für die hintere Extremität von 150 auf $120 \mathrm{~mm}$ R.-A. herabgesetzt (und selbst nach Verabreichung von nur $4 \mathrm{ccm}$ desselben Schlafmittels fällt die Erregbarkeitssch welle von 170 auf $150 \mathrm{~mm}$ R.-A.). Nach Verabreichung von $8 \mathrm{ccm}$ Dormiol sinkt die Erregbarkeitsschwelle für die hintere Extremität von 175 auf $120 \mathrm{~mm}$ R.-A.; nach Verabreichung von $4 \mathrm{ccm}$ Dormiol bei drei Versuchstieren erniedrigte sich im allgemeinen ebenfalls sichtlich die Erregbarkeitsschwelle, wenn auch nicht so auffallend wie bei Amylenhydrat.

Hingegen vermissten wir in unseren Versuchen nach Verabreichung von Veronal und Luminal (selbst in sehr beträchtlichen Dosen) eine unzweifelhafte Herabsetzung der zuvor konstatierten Erregbarkeitsschwelle (vgl. Tab. IV und V). 
Über Erregbarkeit der Grosshirnrinde etc.

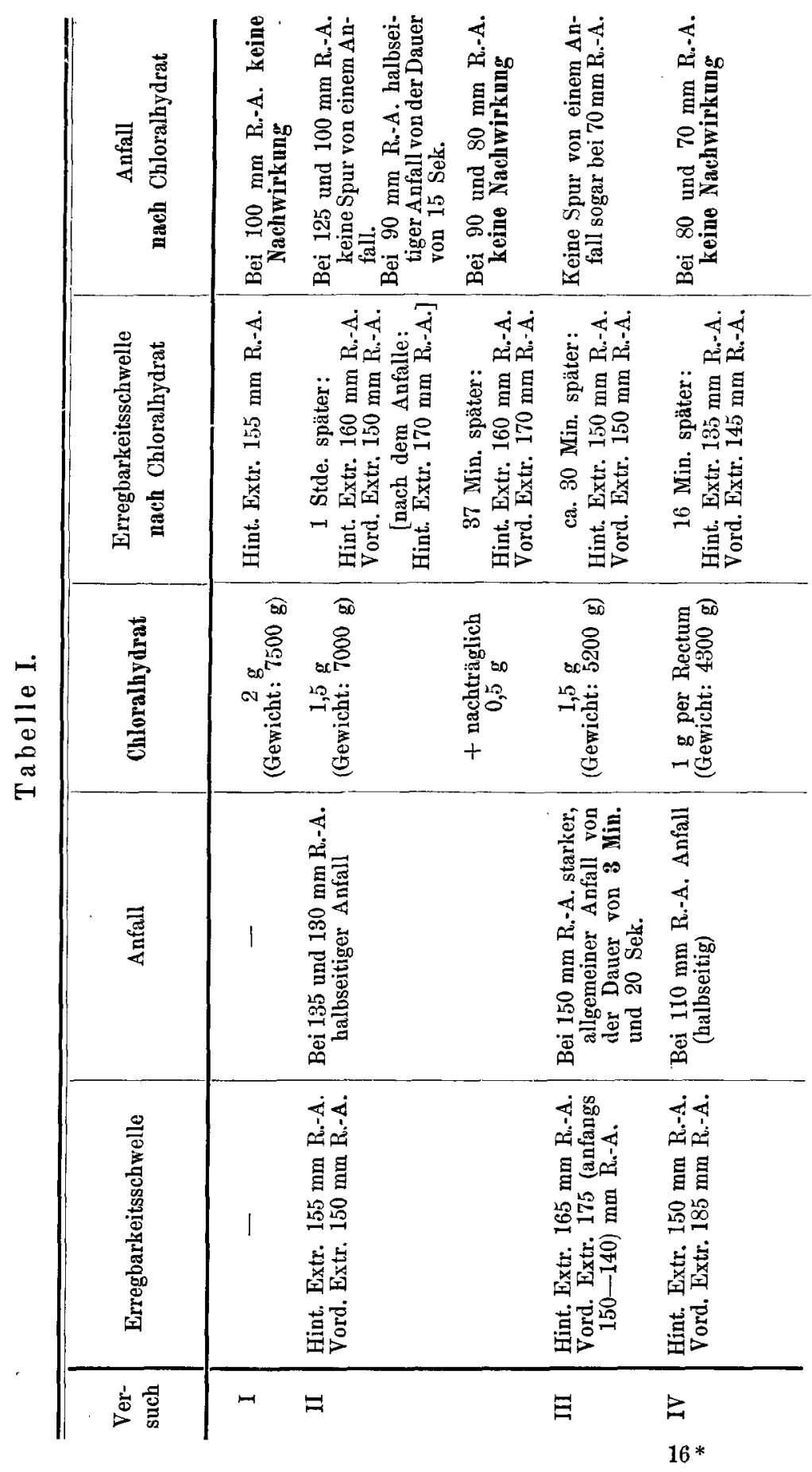


G. Bikeles und L. Zbyszewski:

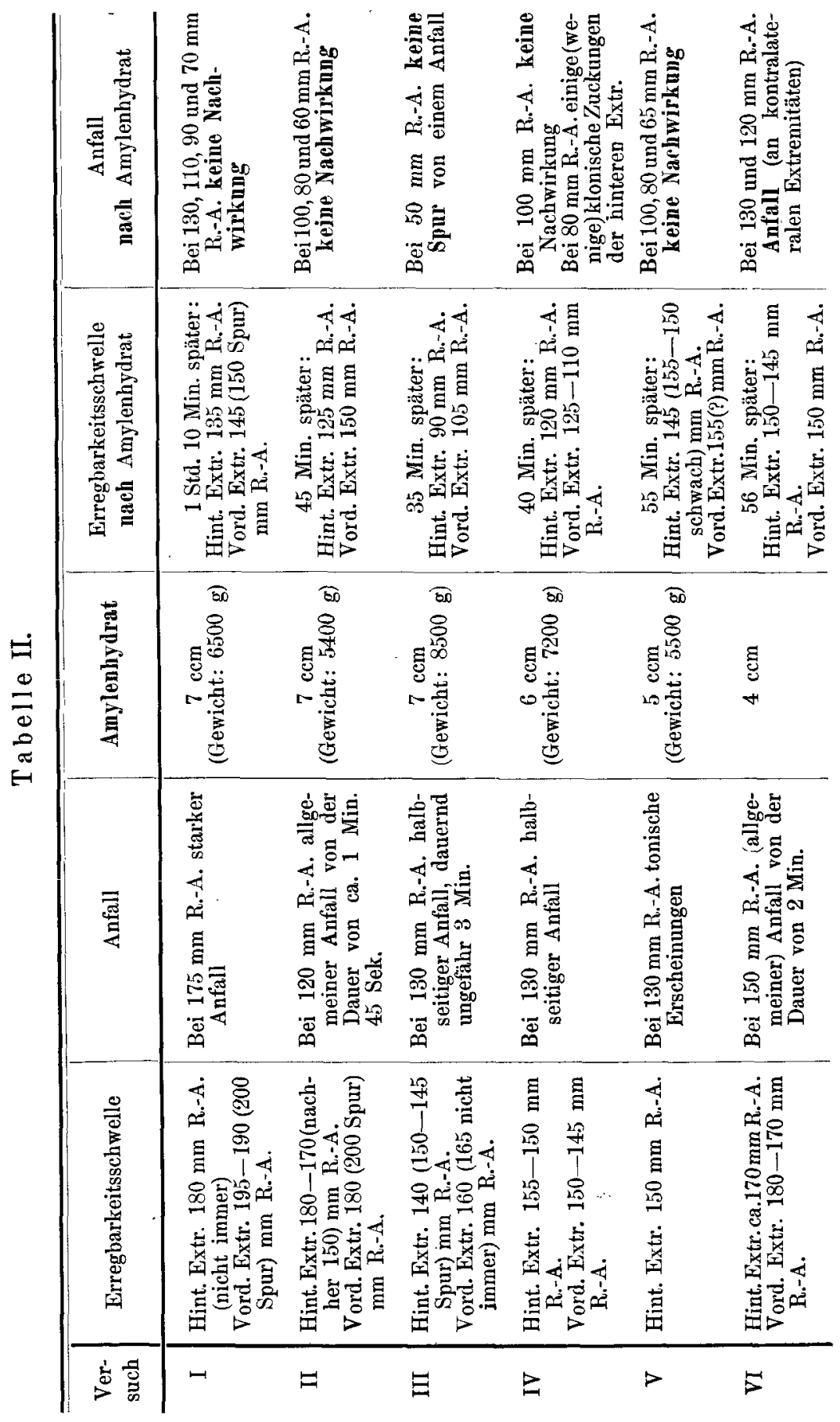


Über Erregbarkeit der Grosshirnrinde etc.

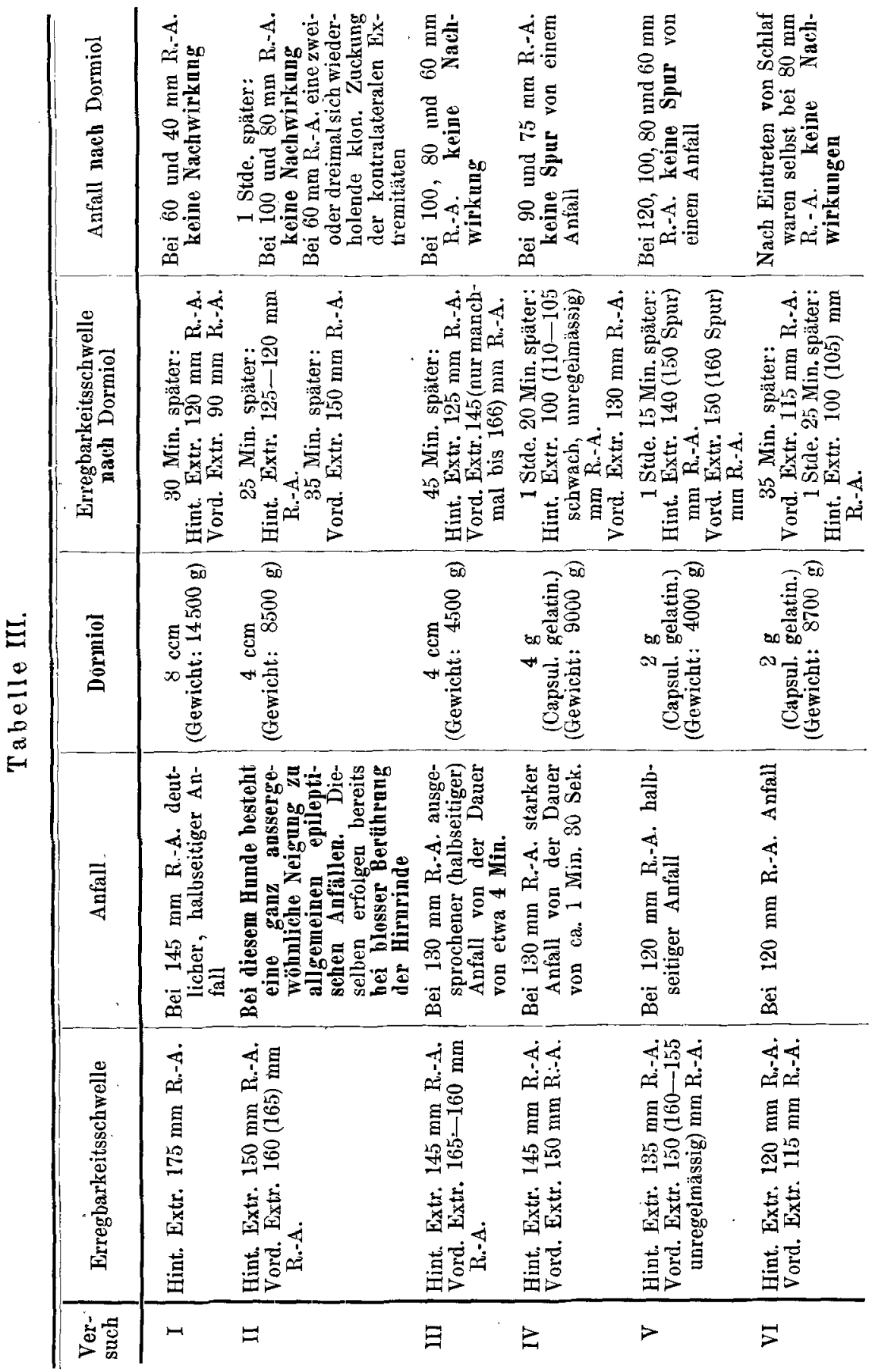




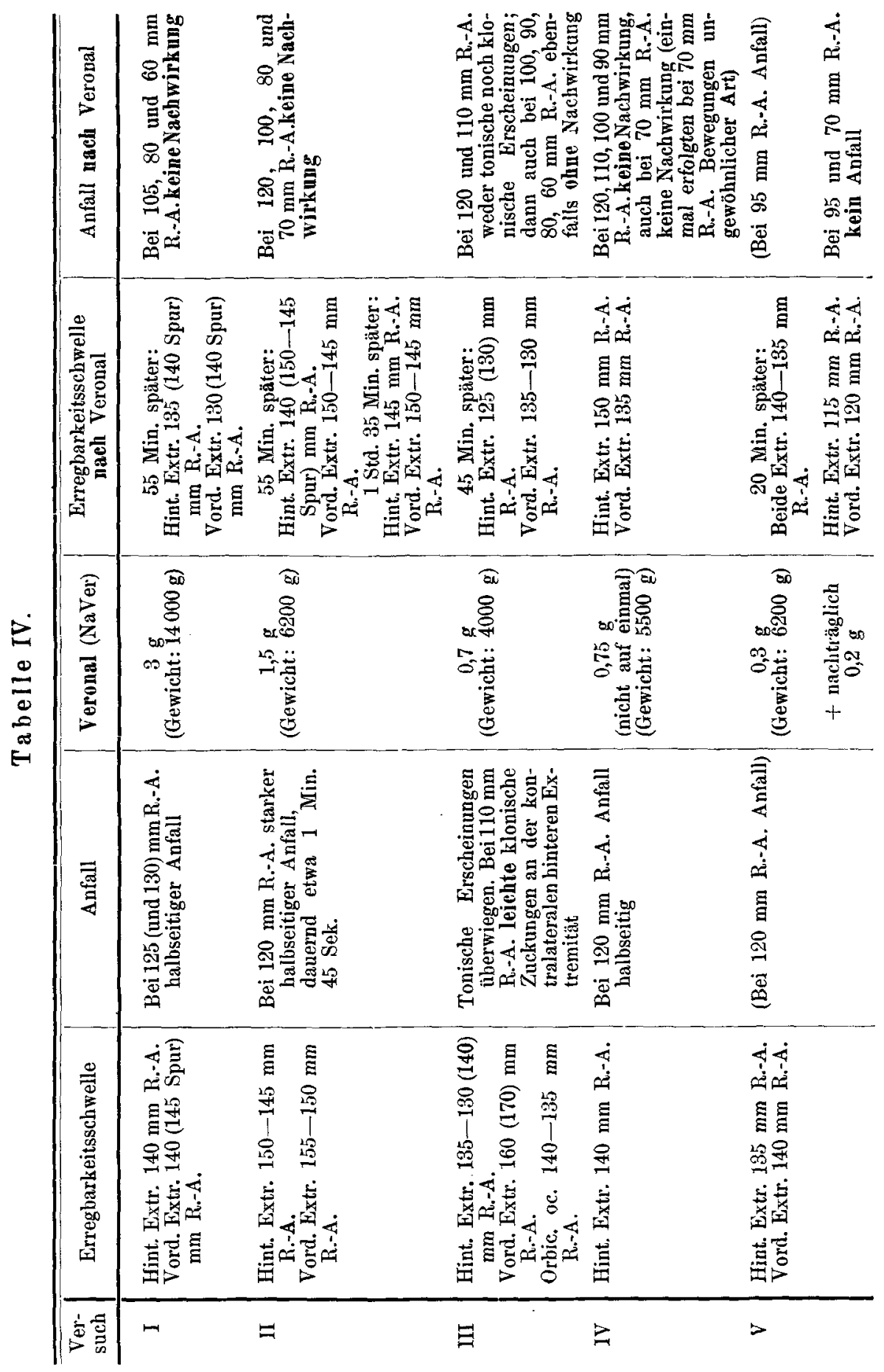


Über Erregbarkeit der Grosshirnrinde etc.
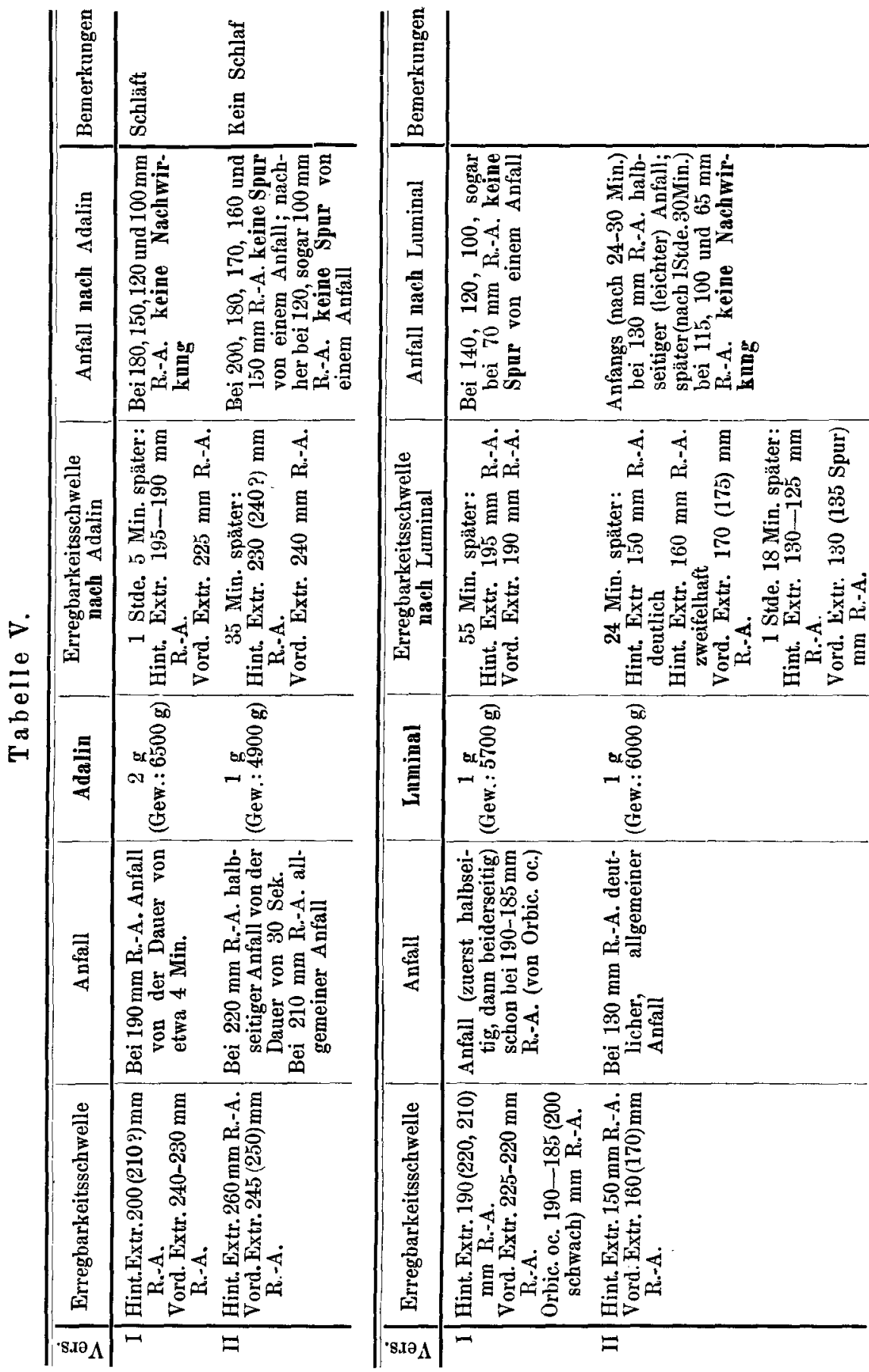


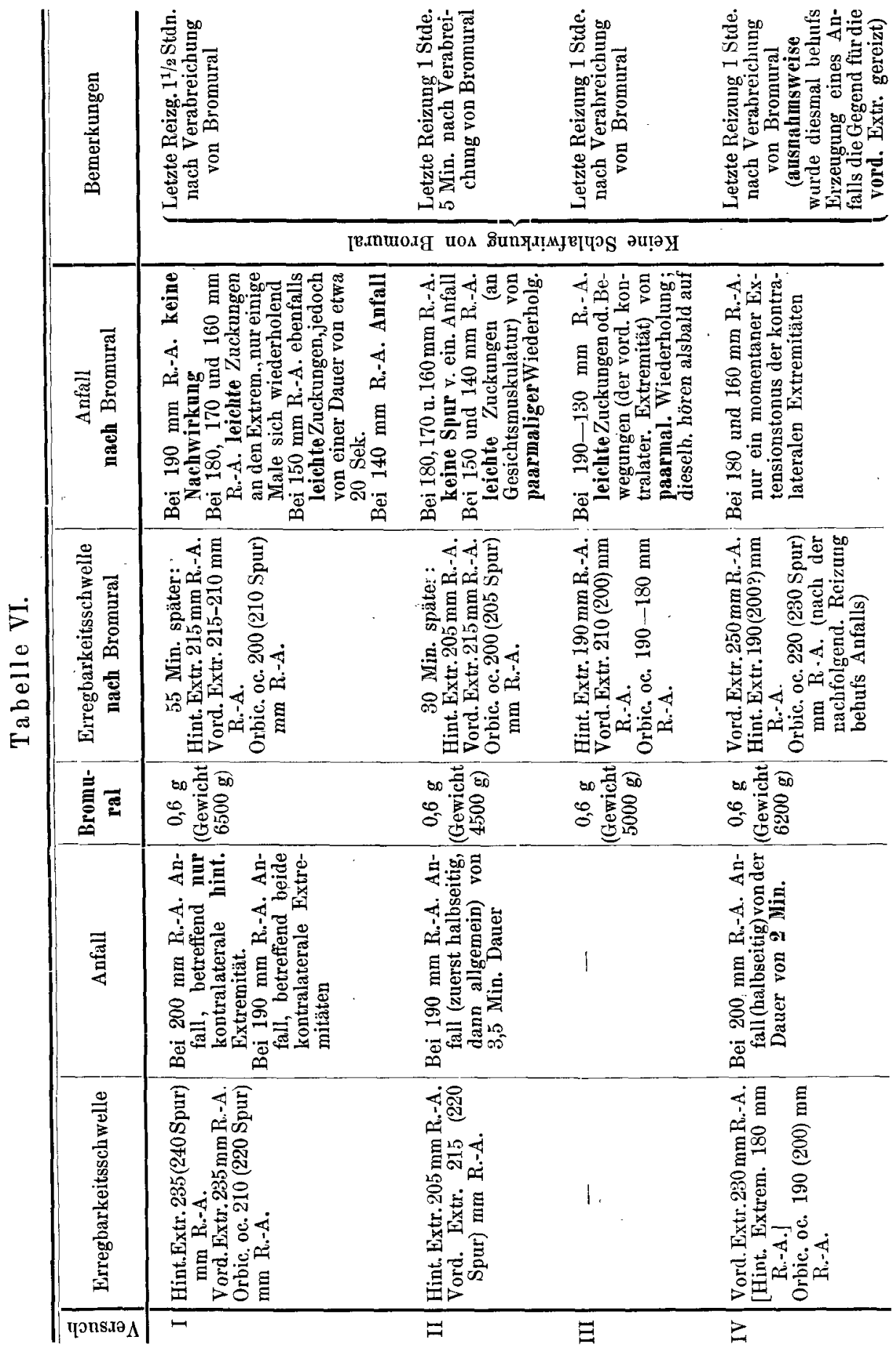


Über Erregbarkeit der Grosshirnrinde etc.
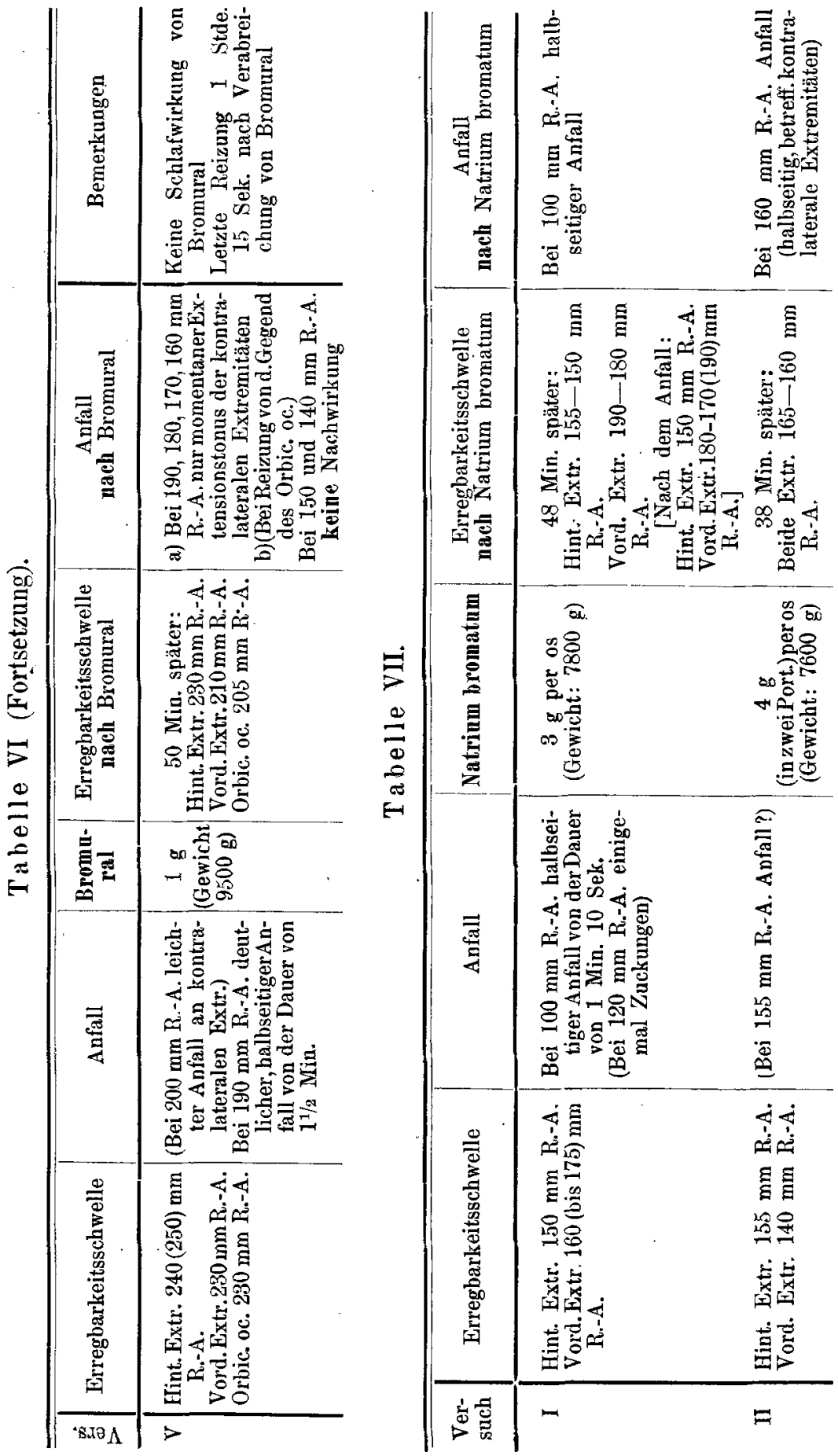


\begin{tabular}{|c|c|c|c|c|}
\hline 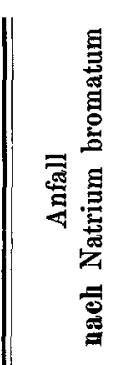 & 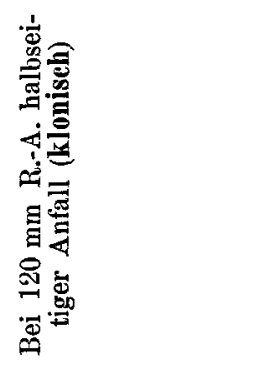 & 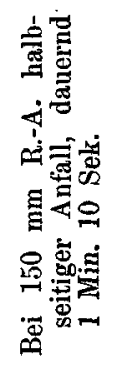 & 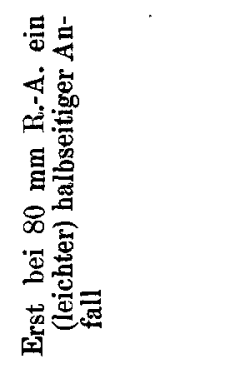 & 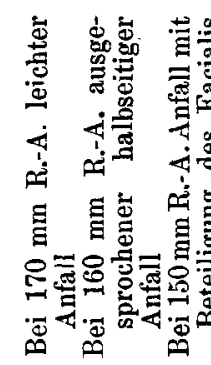 \\
\hline 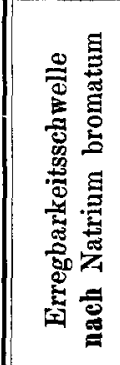 & 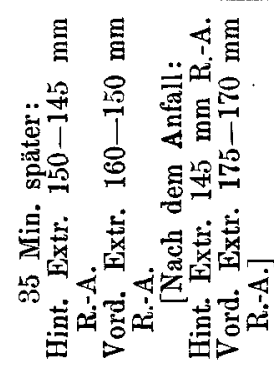 & 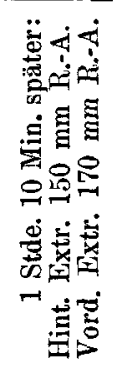 & 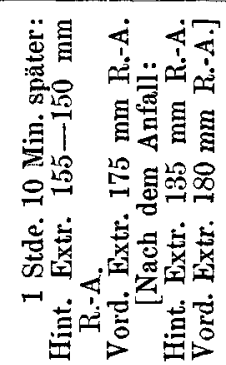 & 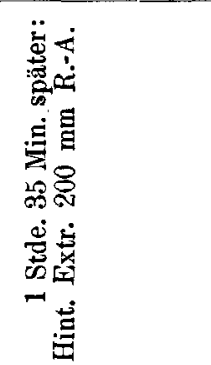 \\
\hline 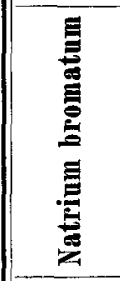 & 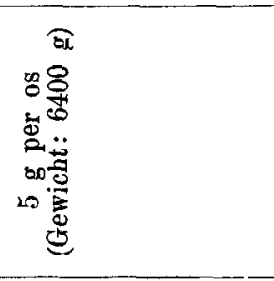 & 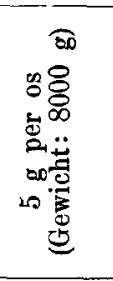 & 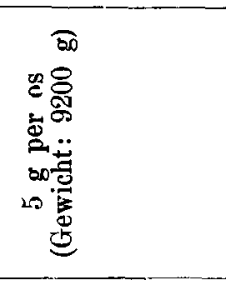 & 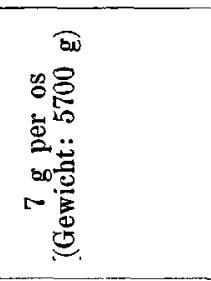 \\
\hline 蹗 & 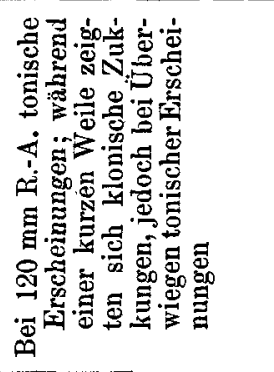 & 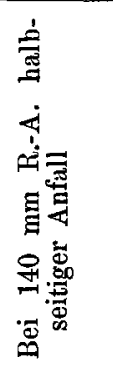 & 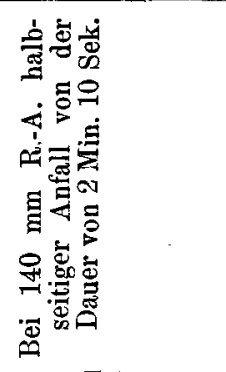 & 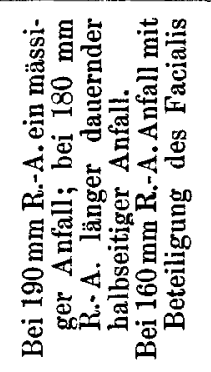 \\
\hline 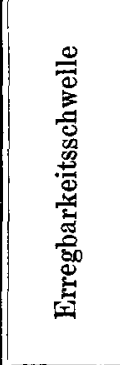 & 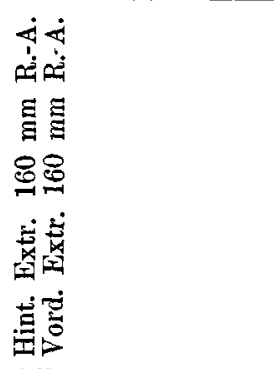 & ن. & 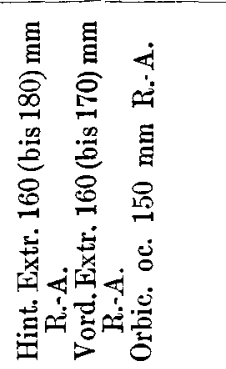 & 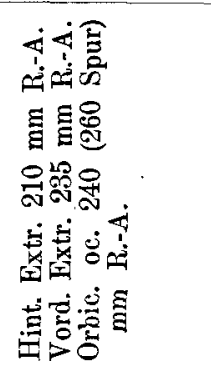 \\
\hline 岀 莺 & $\Xi$ & $\geq$ & $>$ & $\vec{D}$ \\
\hline
\end{tabular}


Über Erregbarkeit der Grosshirnrinde etc.

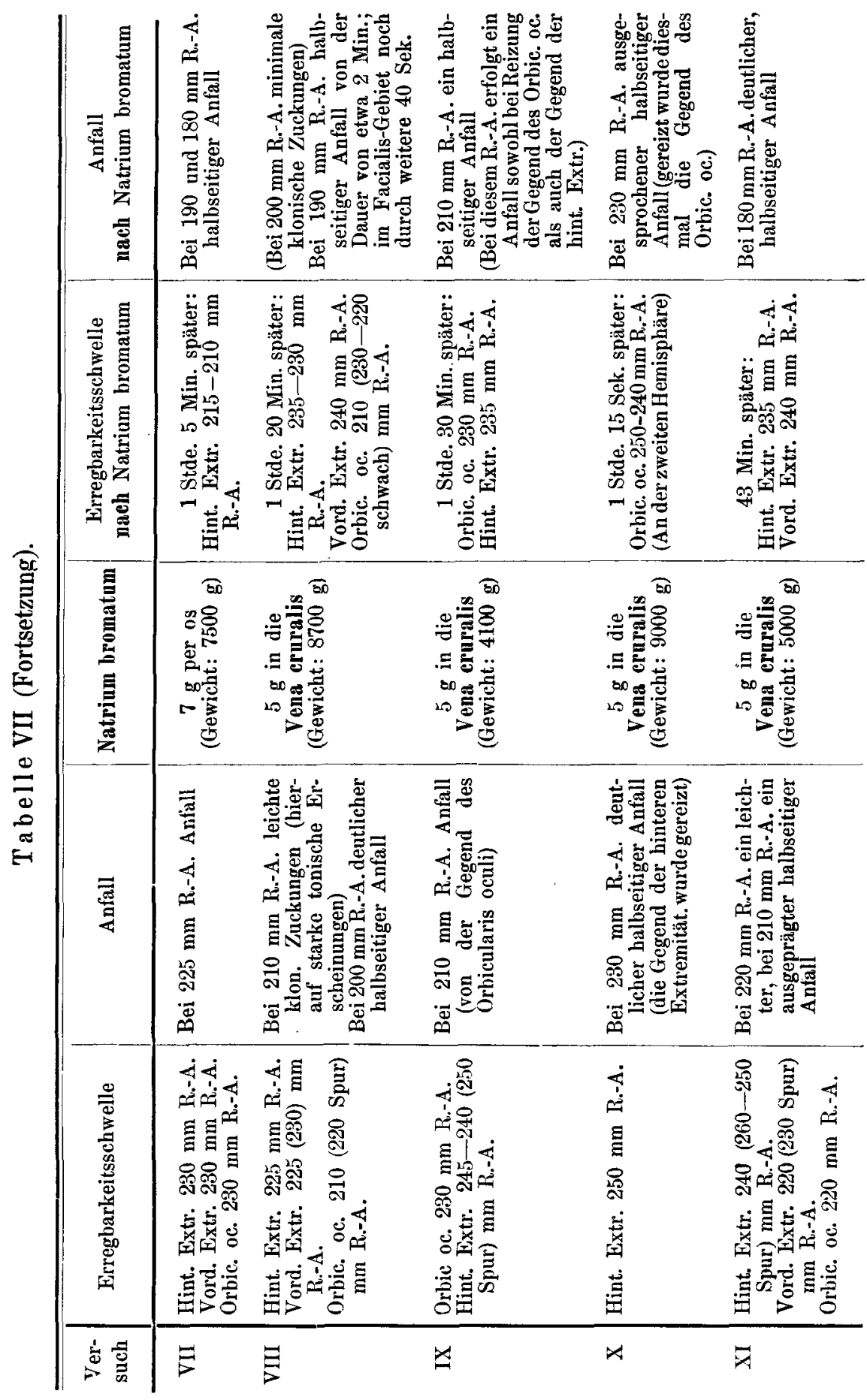




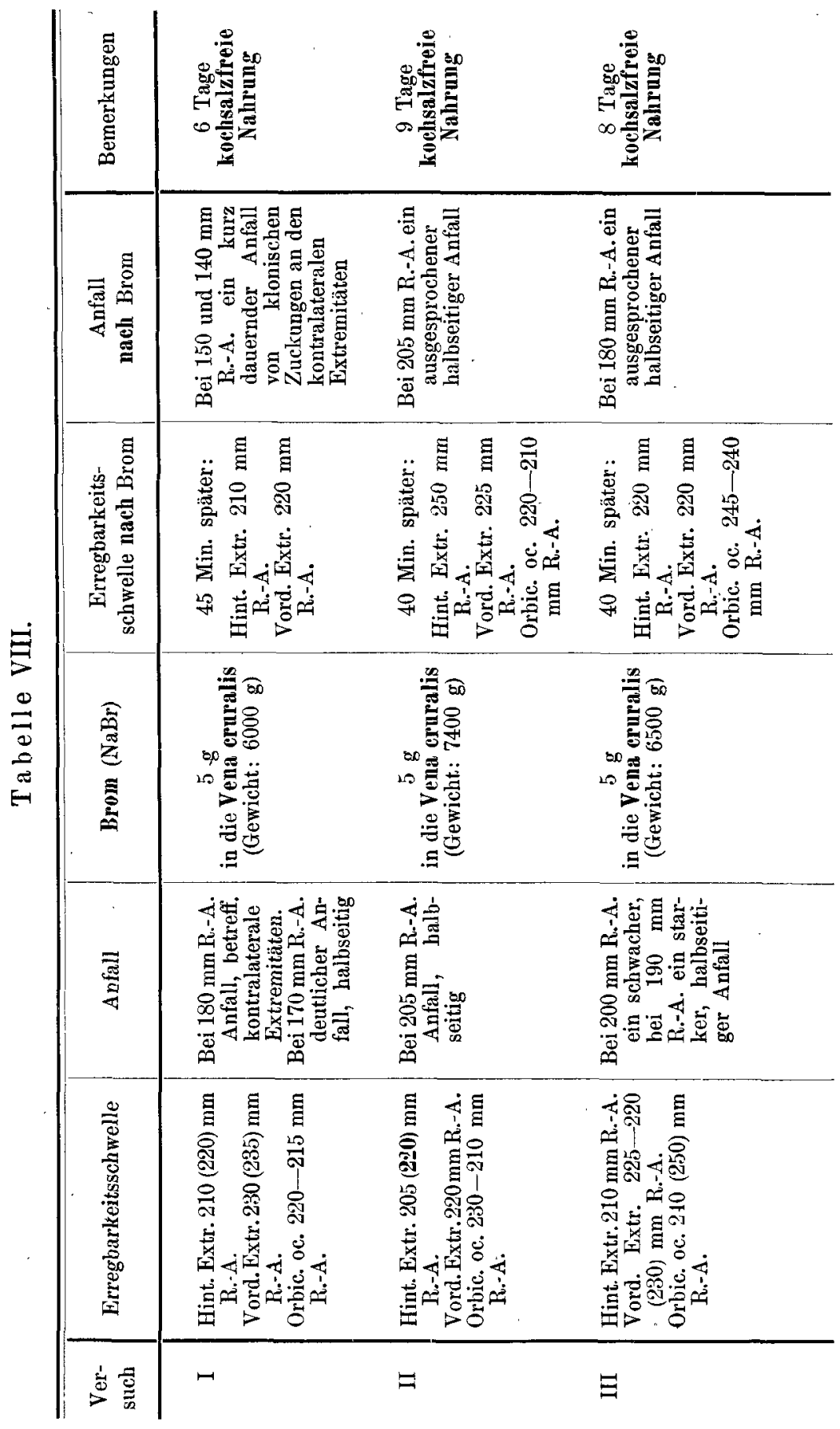


Tabelle IX.

Natrium bromatum und kochsalzfreie Nahrung darch mehrere Tage.

\begin{tabular}{|c|c|c|c|}
\hline$\stackrel{\dot{2}}{\dot{0}}$ & Erregbarkeitsschwelle & Anfall & Natriam bromatam \\
\hline \multirow[t]{2}{*}{ I } & \multirow{2}{*}{$\begin{array}{l}\text { Hint. Extr. } 190 \mathrm{~mm} \text { R.-A. } \\
\text { Vord.Extr. } 180 \mathrm{~mm} \text { R.-A. } \\
\text { Orbic. oculi } 185-180 \mathrm{~mm} \\
\text { R.-A. }\end{array}$} & \multirow{2}{*}{$\begin{array}{l}\text { Bei } 180,170,160,150,140 \text {, } \\
130,120 \text { und } 100 \text { mm R.-A. } \\
\text { kein Anfall (nur ein } \\
\text { Strecktonus der hinteren } \\
\text { Extremität, eventuell ver- } \\
\text { spätet) }\end{array}$} & $\begin{array}{l}\text { Gesamtgabe: Natr. br. } \\
27 \mathrm{~g} .93 \mathrm{~g} \text { täglich } \\
\text { durch } 9 \text { Tage }\end{array}$ \\
\hline & & & $\begin{array}{l}\text { Kochsalzfreie Nahrung } \\
\text { durch } 9 \text { Tage } \\
\text { (Gewicht: } 9000 \mathrm{~g} \text { ) }\end{array}$ \\
\hline \multirow[t]{2}{*}{ II } & \multirow[t]{2}{*}{$\begin{array}{l}\text { Hint. Extr. } 210 \mathrm{~mm} \mathrm{R.-A.} \\
\text { Vord.Extr. } 210 \mathrm{~mm} \text { R.-A. }\end{array}$} & \multirow{2}{*}{$\begin{array}{l}\text { a) Fehlen jeder Spur eines } \\
\text { klonischen Anfalles nach } \\
\text { Reizung bei } 200,190,180 \text {, } \\
170,160,150 \text { und } 140 \mathrm{~mm} \\
\text { R.-A. (dagegen kommt } \\
\text { zum Vorschein eine toni- } \\
\text { sche Verkrümmung des } \\
\text { Rumpfes nach derselben } \\
\text { Seite und ein Strecktonus } \\
\text { der kontralateralen hinte- } \\
\text { ren Extremität). } \\
\text { b) Bei } 130 \mathrm{~mm} \text { R.-A. ein } \\
\text { leichter halbseitiger, klo- } \\
\text { nischer Anfall von der } \\
\text { Dauer von } 42-43 \text { Sek. }\end{array}$} & $\begin{array}{l}\text { Gesamtgabe: Natr. br. } \\
\text { 21 g. } 3 \mathrm{~g} \text { täglich } \\
\text { durch } 7 \text { Tage }\end{array}$ \\
\hline & & & $\begin{array}{l}\text { Kochsalzfreie Nahrung } \\
\text { durch } 8 \text { Tage. } \\
\text { (Gewicht: } 9200 \mathrm{~g} \text { ) }\end{array}$ \\
\hline \multirow[t]{2}{*}{ III } & \multirow{2}{*}{$\begin{array}{l}\text { Hint. Extr. } 215 \mathrm{~mm} \mathrm{R} \text {-A. } \\
\text { Vord. Extr. } 220-210 \mathrm{~mm} \\
\text { R.-A. } \\
\text { Orb. oculi } 225 \mathrm{~mm} \mathrm{R.-A.}\end{array}$} & \multirow{2}{*}{$\begin{array}{l}\text { Bei } 205,190,180,170,160, \\
150 \text { und } 130 \mathrm{~mm} \text { R.-A. } \\
\text { kein Anfall von klo- } \\
\text { nischen Zuckungen (nur } \\
\text { bei } 140 \text { mm R.-A. einige- } \\
\text { mal klonische Zuckungen } \\
\text { der vorderen kontra- } \\
\text { lateralen Extremität, die } \\
\text { aber bald auf hörten). } \\
\text { (Bei } 180-130 \text { mm R.-A. } \\
\text { tonische Erscheinungen } \\
\text { an der hinteren kontra- } \\
\text { lateralen Extremität, } \\
\text { mehr oder weniger deut- } \\
\text { lich.) }\end{array}$} & $\begin{array}{l}\text { Gesamtgabe: Natr. br. } \\
15 \mathrm{~g} .3 \mathrm{~g} \text { täglich } \\
\text { durch } 5 \text { Tage }\end{array}$ \\
\hline & & & $\begin{array}{l}\text { Kochsalzfreie Nahrung } \\
\text { durch } 6 \text { Tage } \\
\text { (Gewicht: } 6700 \mathrm{~g} \text { ) }\end{array}$ \\
\hline
\end{tabular}

Beachtenswert ist ein analoges Verhalten der Erregbarkeitsschwelle für die peripheren Nerven unter Einwirkung derselben Schlafmittel. In einer Reihe unserer Versuche legten wir nämlich den Nervus ischiadicus der einen Seite bloss (auf Seite der gereizten Hirnrinde) und bestimmten den Rollenabstand, bei dem eben deutliche klonische Zuckungen der Extremität bei Reizung des Ischiadicus zum Vorschein kamen; geraume Zeit nach Verabreichung des entsprechenden Schlafmittels wurde abermals der Ischiadicus gereizt und der Rollenabstand bei erfolgter Zuckung der Extremität notiert. 
Es zeigte sich nun, dass bei allen drei Hunden, die je $7 \mathrm{ccm}$ Amylenhydrat erhielten, die Erregbarkeitsschwelle für den Ischiadicus sich ausnahmslos deutlich erniedrigte (von $315-310 \mathrm{~mm}$ auf $275 \mathrm{~mm}$ R.-A., von 300-290 mm auf 260-250 mm R.-A., von $270 \mathrm{~mm}$ auf 230-220 mm R.-A.). Nach Verabreichung von $8 \mathrm{ccm}$ Dormiol sinkt die Erregbarkeitsschwelle für den Nervus ischiadicus von $315 \mathrm{~mm}$ auf $280 \mathrm{~mm}$ R.-A.; nach $4 \mathrm{ccm}$ Dormiol sinkt die Erregbarkeitsschwelle des Ischiadicus einmal von $370 \mathrm{~mm}$ auf $330 \mathrm{~mm}$ R.-A.; beim anderen Hund bleibt sie unverändert. Bei Verabreichung von Veronal erfolgt hingegen (1,5 $\mathrm{g}$ und $3 \mathrm{~g}$ ) gar keine Erniedrigung der Erregbarkeitsschwelle für den Ischiadicus.

Während die Erregbarkeitsschwelle der psychomotorischen Region nur bei gewissen, d. i. den lipoidlösenden Schlafmitteln recht deutlich ausgesprochen ist, heben die Summationsfähigkeit behufs Erzeugung Jacks on'scher Epilepsie auch Veronal, Luminal, sogar Adalin und Bromural auf.

Man könnte von vornherein zur Annahme geneigt sein, dass die Unauslösbarkeit von Rindenepilepsie bei Tieren, die unter dem Einfluss von Schlafmitteln sich befinden, eine der schlaferzeugenden Eigenschaft, also der allgemeinen Herabsetzung der Grosshirnindenfunktion parallele Erscheinung sei. Bei einer derartigen Annahme würde nun die Aufhebung der Auslösbarkeit von Rindenepilepsie nach Verabreichung erwähnter Schlafmittel nur beim Eintreten einer Schlafwirkung zu erwarten sein. Dies trifft aber keinesfalls zu. Schon die bei manchen Versuchstieren verabreichte Gabe von Veronal war bezüglich Schlafwirkung eine verhältnismässig geringe. Noch auffallender ist das Verhalten in unseren Versuchen mit Bromural; bei fünf Versuchstieren, bei denen wir Bromural verabreichten $(0,6$ und einmal $1 \mathrm{~g}$ ), zeigte sich keine Spur von Schlaf, und die Tiere blieben bis zum Schluss des Versuches wach; nichtsdestoweniger aber hörte die Auslösbarkeit von Rindenepilepsie entweder gänzlich auf, oder es wurde dieselbe bedeutend herabgesetzt ${ }^{1}$ ). (Ähnlich wie bei Bromural war das Verhalten nach Verabreichung von $1 \mathrm{~g}$ Adalin.) Man ersieht daraus, dass Schlafwirkung und Unauslösbarkeit von Rindenepilepsie ganz und gar nicht parallel einhergehen, und

1) In einer Reihe späterer Versuche, betreffend Reizwirkung und Lokalisation an der Grosshirnrinde, wandten wir öfters bei Hunden, die eine grosse Neigung zur Rindenepilepsie zeigten, Bromural 0,6 mit sichtbarem Erfolg an. 
dass vielmehr die Summationsfähigkeit der Grosshirnrinde, die zur Erzeugung von Rindenepilepsie nötig ist, schon bei kleineren Gaben, die noch keinen Schlaf erzeugen, herabgesetzt wird. Damit gewinnt die klinische Verwendung von Luminal bei Behandlung der Epilepsie, die in neuester Zeit viele Anhänger gefunden hat, eine theoretische Grundlage.

Ganz anders als nach Schlafmitteln ist das Verhalten nach einmaliger Verabreichung von Brom. Unsere Versuche mit Natrium bromatum lassen sich folgendermaassen gruppieren:

A. bei bisheriger kochsalzhaltiger Nahrung einmalige Bromabgabe, und zwar: a) per os bei sieben Versuchstieren, b) als Injektion in die Vena cruralis bei vier Versuchstieren;

B. bei kochsalzfreier Nahrung (in der Dauer von 6-9 Tagen): a) einmalige Bromgabe als Einspritzung in die Vena cruralis bei drei Versuchstieren, b) per os durch eine Reihe von Tagen bei drei Versuchstieren.

In den Fällen nun von einmaliger Bromdarreichung, gleichviel ob per os oder per venam cruralem, bei zuvor kochsalzhaltiger Nahrung bleibt die Auslösbarkeit von Rindenepilepsie im ganzen genommen nicht wesentlich verändert. Man könnte uns bei einigen (Tab. VII) dieser Versuche bei Darreichung per os die Kürze der Zeit - durch unvorbergesehene Zeitknappheit beim Versuch verursacht - für die abermalige Untersuchung der Erregbarkeitsschwelle bemängeln. Allein selbst in diesen Fällen fanden die Untersuchungen behufs Auslösbarkeit der Rindenepilepsie erst später statt und dauerten auch, indem wir von den schwächsten zu wirksamen stärkeren Strömen gradatim und in Pausen fortschritten, ziemlich lange. Ausserdem wurde in der weit überwiegenden Anzahl der Versuche die abermalige Prüfung der Erregbarkeitsschwelle - wie aus der Tabelle ersichtlich - viel später vorgenommen.

In den Versuchen mit Einspritzung von Natriumbromat in die Vene nach vorausgegangener kochsalzfreier Nahrung ist die Wirkung bezüglich Auslösbarkeit von Jackson'scher Epilepsie ebenfalls keine überzeugende; eine deutlichere Herabdrückung in der Auslösbarkeit eines Anfalles ist eigentlich nur in einem Falle (auf 3) vorhanden, also ein recht zweifelhaftes Ergebnis.

Kann man auch nicht das Ergebnis von einer Wirksamkeit oder Unwirksamkeit einer Substanz bei einer gewissen Dosis direkt vom Hund auf den Menschen übertragen, so ist doch immer der Kontrast 
zwischen dem Verhalten nach Natriumbromat einerseits und Bromural andererseits sehr auffallend.

Bromural, in den relativ kleinen Dosen von $0,6 \mathrm{~g}$ per os gereicht, setzt die Auslösbarkeit der Rindenepilepsie sehr bedeutend herab, resp. hebt dieselbe ganz auf; Natriumbromat dagegen in Gaben von $5 \mathbf{g}$, direkt in die Blutbahn gebracht, bleibt ohne Wirkung. Wir meinen daher, dass eine einmalige Darreichung von Natriumbromat, wenigstens in der von Ärzten gewöhnlich verordneten Dosis von 2-3 $\mathrm{g}$, auch beim Mensehen kaum als wirkliches Sedativum gelten kann.

Natriumbromat in mässiger Gabe zeigte sich in unseren Versuchen nur dann von Einfluss auf die Grosshirnrinde, wenn diese Gabe - selbst per os - durch mehrere Tage (von 5-9) verabreicht worden war (Tab. IX). Während wir bei den übrigen Versuchen mit ganz winzigen Ausnahmen die Erregbarkeit der Hirnrinde und Auslösbarkeit von Jackson'scher Epilepsie vor Anwendung der entsprechenden Substanz und nach Darreichung derselben abermals prüften, haben wir in der letzten Gruppe unserer Versuche die vorhergehende Untersuchung unterlassen. Nichtsdestoweniger überzeugt das übereinstimmende Resultat bezüglich der Nichtauslösbarkeit resp. der bedeutenden Herabsetzung der Auslösbarkeit von Rindenepilepsie nach mehrtägiger Wiederholung mässiger Gaben von Natriumbromat, dass das Fehlen von klonischen Zuckungen als Nachwirkung wirklich die Folge des in dieser Weise dargereichten Natriumbromatsist.

Fragt man, weshalb mässige Gaben von Natriumbromat, durch eine Reihe von Tagen dargereicht, sich wirksamer zeigen, so wäre man von vornherein geneigt, in der grösseren verabreichten Gesamtmenge eine befriedigende Frklärung zu finden. Infolge von Retention von Brom bei tagelanger Darreichung erfolgt jedenfalls eine grössere Anhäufung desselben im Organismus. Wir sind aber der Meinung, dass ausser der Retention von Brom auch noch die Ausscheidung von Chlor [vgl. die Arbeiten von $\left.\mathrm{Wy} \mathrm{s}^{1}\right)$ ] eine grosse Rolle spielt. Dafür spricht das Verhalten in einem nachträglich ausgeführten Versuch. Bei einem Hunde von $5,5 \mathrm{~kg}$ Gewicht injizierten wir in die Vena cruralis eine Lösung von 20\% Natriumbromat, zunächst in der Menge von $10 \mathrm{~g}$ Natrii bromati. Nach entsprechender Pause (etwa 25 Minuten) erhielten wir nach Reizung, 30 Sekunden lang,

1) Arch. f. exper. Path. u. Pharm. Bd. 55 S. 263 und Bd. 59. 
beim Rollenabstand $200 \mathrm{~mm}$ einen heftigen, sehr lange dauernden ( $4^{1 / 2}$ Minuten) allgemeinen epileptischen Anfall, der nach Aufhören sich noch einmal mit ebensolcher Intensität wiederholte. Nachher wurden demselben Hunde noch $5 \mathbf{g}$ Natriumbromat als $20 \%$ ige Lösung: in die Vena cruralis eingespritzt. Nach einer Pause von über $1 / 2$ Stunde wurde die Hirnrinde abermals gereizt, und es resultierte bei $170 \mathrm{~mm}$. R.-A. ein deutlicher, den Facialis ebenfalls betreffender, halbseitiger epileptischer Anfall, der 1 Minute dauerte.

Trotzt also einer direkt in die Blutbahn eingeführten Gabe von. $15 \mathrm{~g}$ Natriumbromat ist die Auslösbarkeit von Rindenepilepsie noch. ausgesprochener vorhanden als in den Fällen, in denen wir Natriumbromat durch eine Reihe von Tagen per os einführten. In letzteren. Fällen betrug die Gesamtmenge durch 5 resp. 7 Tage 15 und $21 \mathrm{~g}$ Bromnatrium; die zuräckgehaltene Menge war jedenfalls kleiner. Dabei war das Körpergewicht der Versuchstiere beträchtlich bedeutender als in dem nachträglich ausgefuhrten Versuch.

Dies weist also darauf hin, dass für die Beeinträchtigung der Funktion der Nervenzelle neben der Anhäufung von Brom im Bluteauch die Verminderung des Chlorgehaltes ${ }^{1}$ ) in Betracht kommt.

\section{Zus ammenfassung.}

1. Herabdrückung oder Aufhebung der Auslösbarkeit von Rindenepilepsie geht nicht immer parallel mit einer Veränderung. der Erregbarkeitsschwelle einher.

2. Die Unauslösbarkeit von Rindenepilepsie infolge der Verabreichung von Hypnotika ist nicht notwendig der Ausdruck einer Aufhebung der Rindenfunktion überhaupt oder proportional der Schlafwirkung, sondern zeigt sich bereits bei zur Schlafwirkung nicht ausreichender Dosis im vollständig wachen Zustand des Tieres.

3. Brom in relativ noch mässiger Dosis als e inmalige Gabe, sogar als Einspritzung in die Vene, bleibt ohne Erfolg auf Erregbarkeit wie auf die Auslösbarkeit von Rindenepilepsie.

4. Mässige Bromgaben zeigen sich wirksam bei Verabreichung: derselben durch eine Reihe von Tagen.

1) Vgl. Wyss, l. c., dann Laudenheimer, Neurol. Zentralbl. 1910 S. 461, und Ulrich, Neurol. Zentralbl. 1910 S. 74. 